\title{
EXPERIMENTAL INVESTIGATIONS OF ELIMINATION THE STICK-SLIP PHENOMENON IN THE PRESENCE OF LONGITUDINAL TANGENTIAL VIBRATION
}

\author{
Mariusz LEUS*, Marta ABRAHAMOWICZ* \\ *Department of Mechanical Engineering and Mechatronics, West Pomeranian University of Technology in Szczecin \\ al. Piastów 19, 70-310 Szczecin, Poland \\ mariusz.leus@zut.edu.pl; marta.abrahamowicz@zut.edu.pl
}

received 2 January 2019, revised 13 March 2019, accepted 20 March 2019

\begin{abstract}
The article presents a scheme and description of the test stand as well as selected experimental results of the influence of longitudinal tangential vibrations on the stick-slip phenomenon. The tests were carried out at a constant forced vibration frequency $f=2000 \mathrm{~Hz}$, as a function of the amplitude of the vibration velocity $v_{a}$. The position of the sliding body and the drive force necessary to make the body slip and maintain this motion were measured. The measurements were made in two successive stages. In the first stage, when the substrate on which the sliding occurred was stationary. In the second one, the substrate is in a vibrating motion in the direction parallel to the slip. The conducted experimental analyses have shown that longitudinal tangential vibrations can contribute to the reduction or even complete elimination of the stick-slip phenomenon.
\end{abstract}

Key words: stick-slip phenomenon, drive force, longitudinal tangential vibrations

\section{INTRODUCTION}

Uncontrolled stepwise movements of the sliding body, known as the stick-slip phenomenon, which can occur in the sliding motion as a result of the friction force, are an undesirable phenomenon and hinder proper operation of the machines. The main cause of their formation is attributed to high difference between static and kinetic friction coefficients (Abdo et al., 2010; Abdo and Zaier, 2012; Canudas de Wit et al., 1995; Mfoumou et al., 2019; Mokhtar et al., 1998; Neubauer et al., 2005), increase in static friction force during standstill, low slip velocity, or low stiffness of the friction pair system (Ozaki and Hashiguchi, 2010).

This unstable movement is repeated at short intervals, more or less regularly, until the slip velocity of the sliding body does not exceed the so-called critical velocity. Above this speed, only motion of the body appears, which is deprived of undesirable jumps. Therefore, the amplitude and frequency of the stick-slip motion depend mainly on the sliding velocity. The mass of the sliding element and the stiffness of the system also play an important role (Grudziński and Warda, 1993).

The stick-slip motion is a phenomenon that occurs both in everyday life and in technology. The effect of this phenomenon is the sound of a bow on the strings of a violin, the squeaking sound of hinged doors, the squeak of tram wheels on a turn and the squeak of chalk on the board (Abdo et al., 2010; Kligerman and Varenberg, 2014; Popp and Rudolph, 2004). The stick-slip phenomenon may also cause disturbances in the movement of moving assemblies of machine tools, positioning devices, measuring instruments or manipulators (Mfoumou et al., 2019; Rymuza, 1992). This has a negative effect on the positioning accuracy of the components, as their position is determined by the amplitude of longitudinal vibrations - the amplitude of jump of the sliding element. The stick-slip vibrations are also common in the drilling operations in the oil and gas industry. They drastically decrease the rate of penetration (Qiu et al., 2018; F. Aarsnes et al., 2018), cause premature tool failures and adversely affect the borehole quality (Tang et al., 2015, 2017), which significantly increases drilling costs (Zhu et al., 2014).

The stick-slip motion contributes to faster wear of the friction pair's components. Therefore, these vibrations may be acceptable, provided that their amplitude is sufficiently small (Popp and Rudolph, 2004). However, it is desirable to completely eliminate or at least reduce the intensity of amplitude of these vibrations. There are several ways to eliminate the stick-slip phenomenon, which include selection of optimal materials and surface roughness of friction pair elements (Kligerman and Verenberg, 2014; Kröger et al., 2008), application of lubrication (Zuleeg, 2015), reduction of normal pressure at the contact (Kröger et al., 2008), replacement of sliding contact with rolling contact (Rymuza, 1992) and introduction of forced external vibrations into the area of contact, which cause a reduction of the time of standstill of the sliding body (Abdo et al., 2009, 2010; Abdo and Zaier, 2012; Neubauer et al., 2005; Popov et al., 2010; Popp and Rudolph, 2003, 2004; Teidelt et al., 2012).

In the works available in the literature, in which the issue of the influence of vibrations on the stick-slip phenomenon was discussed, the main attention has so far been focused on the use of normal vibrations to eliminate this phenomenon. In these works, two ways of introducing vibrations into the contact area were presented. Abdo et al. (2009, 2010) and Abdo and Zaier (2012) carried out the experimental tests of stick-slip phenomenon on the especially constructed pin-on-disc machine, which gives the possibility to generate vertical vibrations of revolving disk. The obtained results show that the level of stick-slip amplitude reduction depends on factors such as the type of friction pair material, amplitude and frequency of excited vibrations and relative humidity. The authors of the above-mentioned work observed that with the 
increase in amplitude of excited vibration, the amplitude of stickslip motion significantly decreased. Neubauer et al. (2005) and Popp and Rudolph $(2003,2004)$ described the possibility of stickslip reduction through the active control of vibration with the use of oscillating normal force. The tests carried out by them confirmed that after active control, the stick-slip motion disappeared.

In the recent years, works in which the results of experimental research indicate the possibility of using tangential vibrations to reduce the stick-slip movement have also appeared (Popov et al., 2010; Teidelt et al., 2012).

This article presents a test stand and selected results of experimental tests of the application of tangential vibrations of the substrate on which the slip movement is performed to the elimination of the stick-slip phenomenon. Experimental tests were carried out as a function of the amplitude of vibration velocity $v_{a}$ at their determined frequency $f$ and determined drive velocity $v_{d}$. The direction of tangential vibrations of the substrate was assumed to be in line with the direction of sliding of the body. The tests were performed for the steel-steel contact.

\section{DESCRIPTION OF THE STICK-SLIP PHENOMENON}

The physical model of a sliding pair with kinematic inducement for the analysis of the stick-slip phenomenon is shown in Fig. 1. The model consists of a body $\boldsymbol{A}$ of mass $m$ sliding on a substrate $\boldsymbol{B}$ by means of a drive providing a constant velocity $v_{d}$. The sliding body is connected with the drive by means of a spring with $a$ stiffness of $k_{d}$.

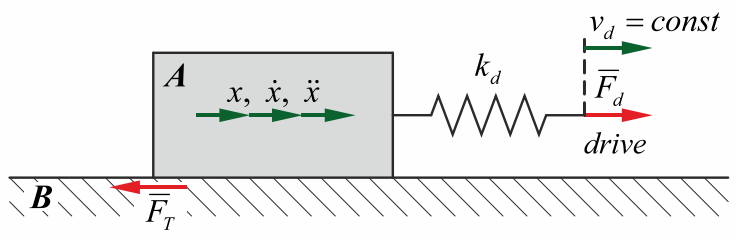

Fig. 1. Physical model of the sliding pair

The equation of the motion of the sliding body $\boldsymbol{A}$ along the axis of the assumed direction of sliding in accordance with the direction of the drive is in the following form:

$m \ddot{x}=F_{d}-F_{T}$,

where $m$ is the mass of the sliding body, $\ddot{x}$ is the its acceleration, $F_{d}$ is the drive force and $F_{T}$ is the friction force.

The drive force can be determined from the following dependence:

$F_{d}=k_{d}\left(s_{d}-x\right)$,

where

$s_{d}=v_{d} \cdot t$.

The $k_{d}$ value is the drive stiffness, $s_{d}$ is the drive displacement, $x$ is the position of the sliding body, $v_{d}$ is the drive speed and $t$ is the time.

The characteristics representing the course of the stick-slip phenomenon in the form of rest periods of body $\boldsymbol{A}$ (stick $-t_{s}$ ) and slip-jump (slip $-t_{r}$ ), corresponding to the model of the slide pair shown in Fig. 1 are schematically presented in Fig. 2.

In the time interval from $t=0$ (the moment when the drive starts to move at $v_{d}=$ const) to $t=t_{s 1}$, body $\boldsymbol{A}$ is at rest, because the drive force $F_{d}$ is lower than the force of static friction $F_{T O}=$ $\mu m g$, where $\mu$ is the friction coefficient and $g$ is the gravitational acceleration. After reaching the time $t_{s 1}$, the drive force $F_{d}$ starts to exceed the friction force $F_{T 0}$ and the slip movement of the body $A$ starts with high acceleration $\ddot{x}$ and its slip speed $\dot{x}$ exceeds the value of the drive speed setpoint $v_{d}$. After half of the duration of motion $t_{r 1}$ has elapsed, the value of the drive force $F_{d}$ decreases below the value of the friction force $F_{T}$ and the braking of the sliding body begins, which after reaching the time $t_{r 1}$ stops and remains at rest during the period $t_{s 2}$. After this time, the movement of the sliding body is repeated cyclically. In real systems, the effect is a jumping movement of the sliding element, the so-called stick-slip phenomenon.

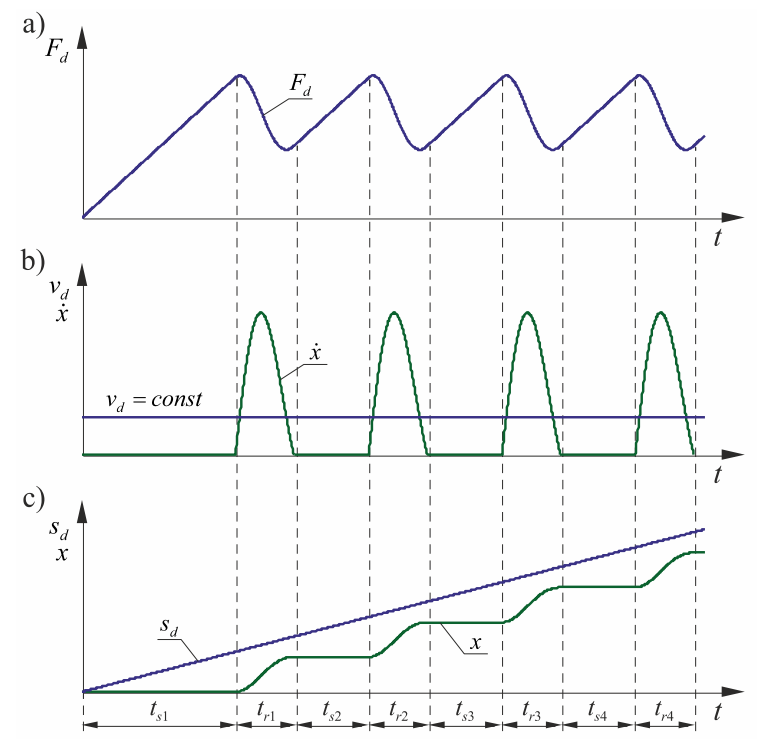

Fig. 2. Characteristics of the stick-slip phenomenon. Time courses of: (a) drive force $F_{d}$, (b) drive velocity $v_{d}$ and velocity of the sliding body $\dot{x}$ and (c) drive displacement $s_{d}$ and position $x$ of the sliding body

\section{THE TEST STAND}

The experimental test of the stick-slip phenomenon in the presence of longitudinal tangential vibrations was carried out on a test stand designed and constructed to determine the quantitative changes in friction forces in the sliding movement caused by the introduction of tangential vibrations into the contact area of the sliding pair. A detailed description of this stand is presented in the works (Leus and Gutowski, 2011; Gutowski and Leus, 2012, 2015). To determine the impact of vibrations on the stick-slip motion, this stand is equipped with an additional system to measure the position of the body and drive and a mechanism to change the stiffness of the drive system. Mechanical part of the test stand prepared for testing is presented in Fig. 3, whereas Fig. 4 shows the block diagram of the elaborated stand.

The basic element of this stand is a sliding pair consisting of a sliding upper sample and a fixed sample placed on the roller guides of the base. As the upper sample is moved, the lower sample can be set in a vibrating motion in accordance with the direction of movement. Vibration is induced by a piezoelectric inductor powered by the PIEZOMECHANIC RCV 1000/3 amplifier. The sliding motion of the upper sample is achieved by a drive system consisting of an EPX40 linear guide equipped with a 
propeller, a step-motor with a gear and a driver. This system also includes a ring dynamometer and a unit with a certain stiffness to transmit the drive force. Replacement of the spring in this assembly allows to change the stiffness of the drive system $k_{d}$ (Fig. 5). The motor operation is controlled by the SMC64v2 controller. The change in velocity $v_{d}$ is obtained by changing the frequency $f_{\text {gen }}$ of impulses fed from the rectangular waveform generator to this controller. In addition, the upper sample may be loaded in the normal direction with an additional external force $F_{z}$ centrally applied to the sample by means of a band loaded with a lever with weights.

During the test, several values, such as the drive force $F_{d}$, the external force $F_{z}$, the acceleration $\ddot{x}$ and the displacement $x$ of the upper sample, the acceleration $\ddot{u}$ of the lower sample, the drive displacement $s$ and the drive speed $v_{d}$, are measured simultaneously. The value of the drive force $F_{d}$ and the external force $F_{z}$ are measured with ring dynamometers, whereas the measure- ment of the distance $x$ travelled by the sliding body and the distance $s$ travelled by the drive is performed with WA-L inductive displacement transducers placed in the sliding axis. Signals from ring dynamometers and displacement transducers are transmitted to the HBM MGA II amplifier equipped with ME10 and ME50 measuring modules. The acceleration of the upper and lower samples is measured by PCB acceleration sensors, which send signals to the PCB 481A load amplifier. To measure the drive speed $v_{d}$, an MOK50 encoder was used, whose rotary axis was connected to the lead screw of the linear guide.

All measured signals are transferred to a measurement computer equipped with a DS1104 measurement card and ControlDesk software from dSPACE. This system provides continuous control and recording of the measured values and enables the control of the vibration inductor amplifier and the step-motor controller.

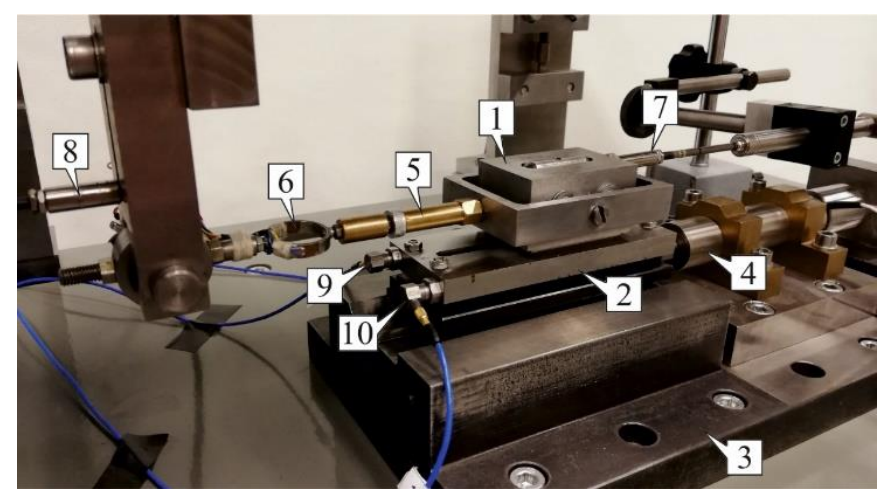

Fig. 3. Photo of mechanical part of test stand: 1 , upper sample; 2 , lower sample; 3 , base; 4 , vibration exciter; 5 , stiffness adjustment system $k_{d} ; 6$, ring dynamometer; 7 and 8, WA-L displacement transducers; 9 and 10, accelerometers

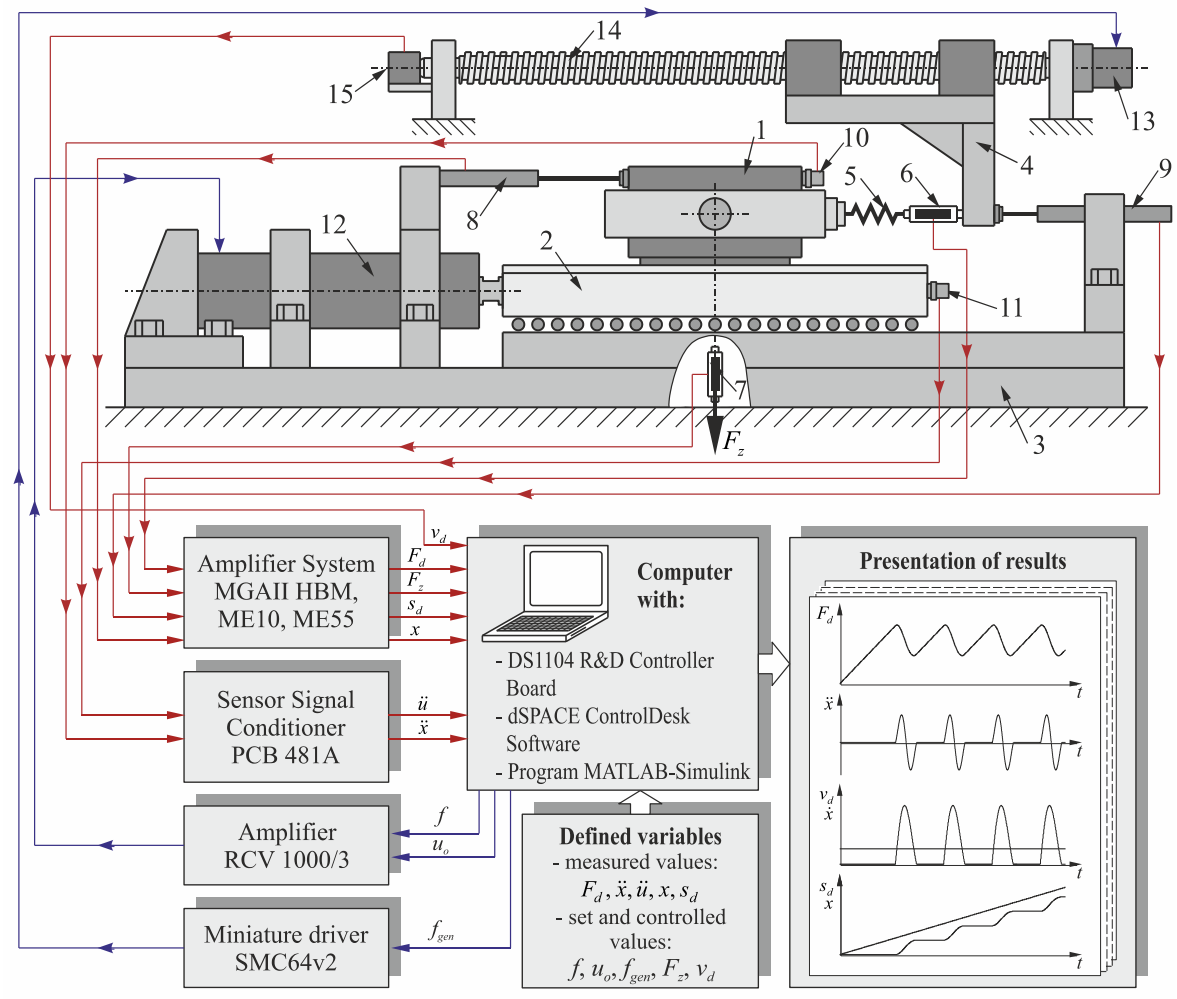

Fig. 4. Diagram of the test stand together with the measurement and recording system: 1 , upper sample; 2 , lower sample; 3 , base; 4 , driver; 5 , stiffness adjustment system $k_{d} ; 6$ and 7 , ring dynamometers; 8 and 9 , WA-L displacement transducers; 10 and 11, accelerometers; 12, vibration exciter; 13 , step-motor with a gear; 14 , linear guide; 15 , encoder 


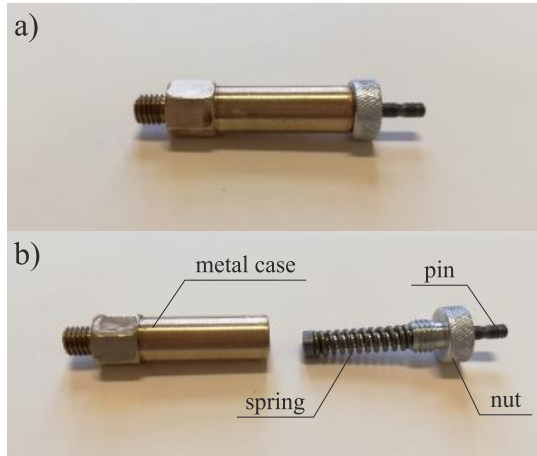

Fig. 5. Drive stiffness $k_{d}$ change system: (a) general view and (b) detailed view

\section{EXPERIMENTAL INVESTIGATIONS AND THEIR OUTCOMES}

The aim of the experimental tests presented in this article was to investigate the possibility of using forced longitudinal tangential vibrations introduced into the contact area to eliminate the stickslip phenomenon. In this study, the quantitative changes the drive force $F_{d}$ and the position of the moved body $x$ in two consecutive stages of motion were determined. In each variant, the movement of the upper body was carried out without forced vibrations in the first stage and with forced vibrations of the lower body in the second stage.
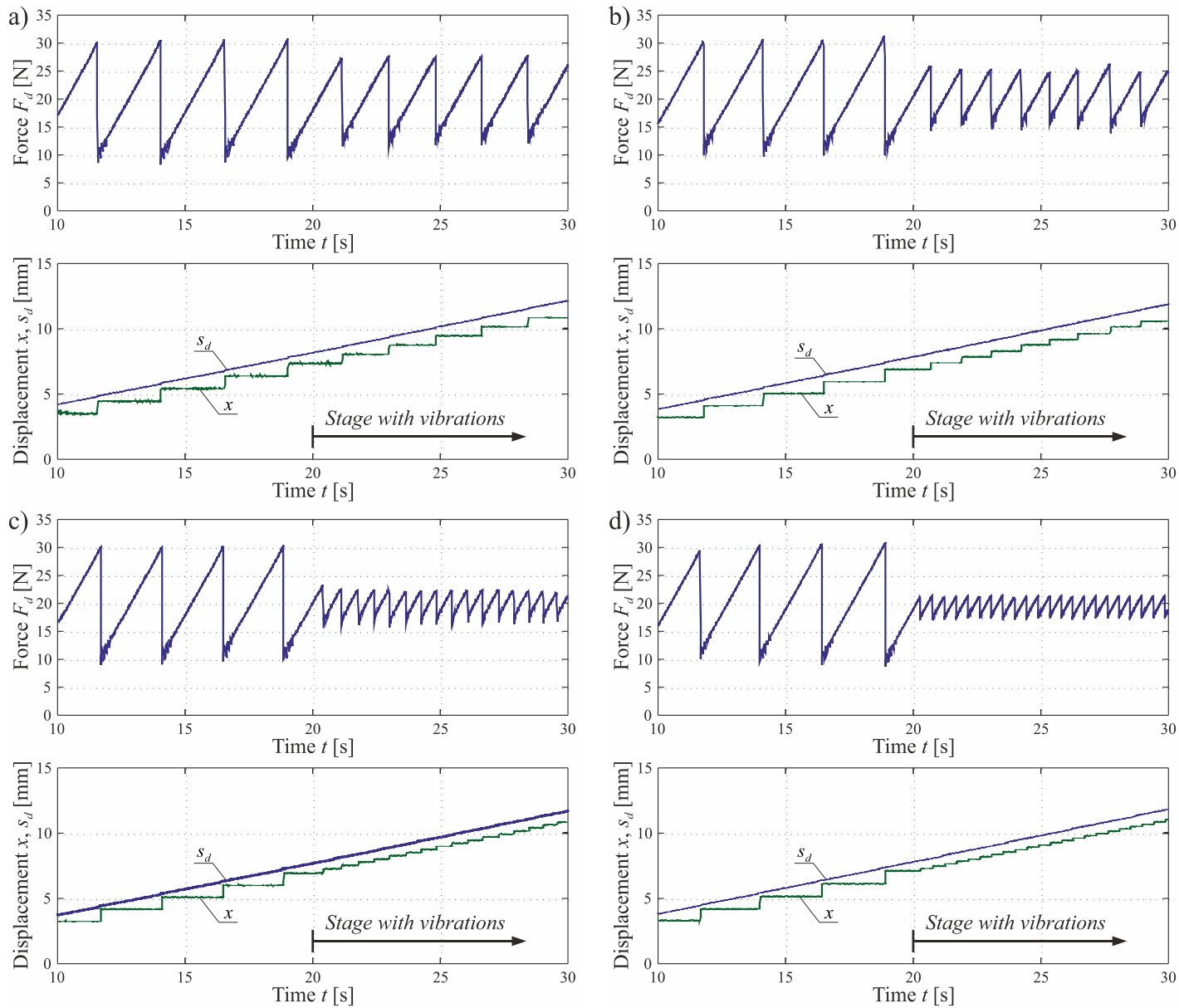

Fig. 6. Experimentally determined waveforms of drive force $F_{d}$, displacement of the sliding body $x$ and drive displacement $s_{d}$ for: (a) $v_{a}=0.1 \mathrm{~mm} / \mathrm{s}$, (b) $v_{a}=0.15 \mathrm{~mm} / \mathrm{s}$, (c) $v_{a}=0.25 \mathrm{~mm} / \mathrm{s}$ and (d) $v_{a}=0.3 \mathrm{~mm} / \mathrm{s} ; f=2000 \mathrm{~Hz}, v_{d}=0.4 \mathrm{~mm} / \mathrm{s}$. 

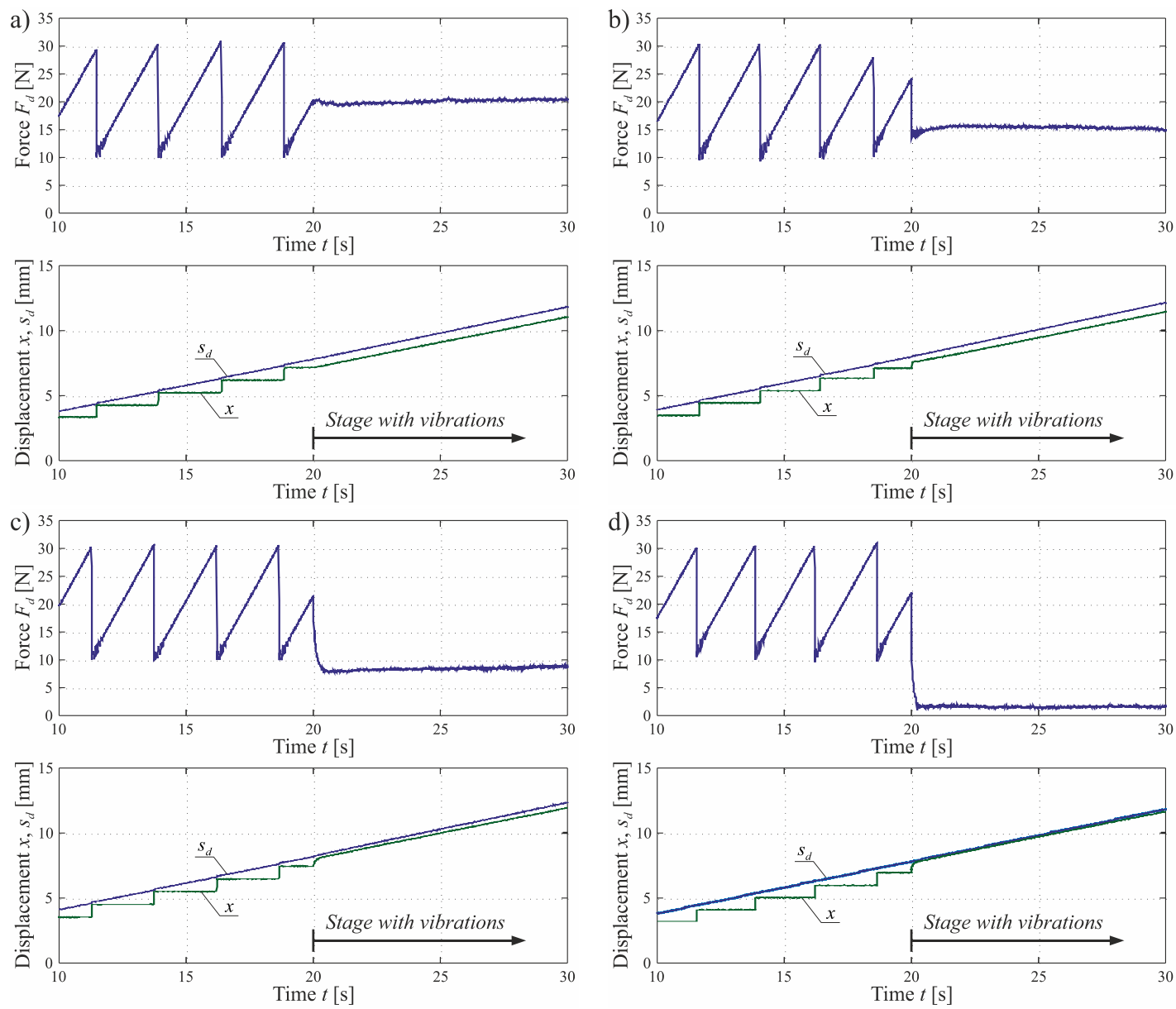

Fig. 7. Experimentally determined waveforms of drive force $F_{d}$, displacement of the sliding body $x$ and drive displacement $s_{d}$ for: (a) $V_{a}=0.4 \mathrm{~mm} / \mathrm{s}$, (b) $v_{a}=0.8 \mathrm{~mm} / \mathrm{s}$, (c) $v_{a}=2 \mathrm{~mm} / \mathrm{s}$ and (d) $v_{a}=6 \mathrm{~mm} / \mathrm{s} ; f=2000 \mathrm{~Hz}, v_{d}=0.4 \mathrm{~mm} / \mathrm{s}$

In the second variant, presented in Fig. 7, the amplitude $v_{a}$ of the vibration velocity was equal to (Fig. 7a) and higher (Fig. $7 b, c, d)$ than the drive velocity $v_{d}$. Also, in this case, the tests were performed for four selected $v_{a}$ values amounting to $0.4,0.8,2$, and $6 \mathrm{~mm} / \mathrm{s}$. From the presented waveforms of the drive force $F_{d}$ and the position $x$ of the sliding body, it can be seen that, in this variant of tests, the stick-slip phenomenon has been completely eliminated.

In addition, in three cases (Fig. 7b,c,d) where va was higher than the drive speed $v_{d}$, the induction of tangential vibrations also caused a significant reduction in the drive force necessary to make the body move in a sliding motion and to maintain this movement. This relationship is also confirmed by the results of experimental research by Leus and Gutowski (2011) and Gutowski and Leus (2012) and by other authors (Gao et al., 2016; Kapelke and Seemann, 2018; Littman et al., 2001a, 2001b; Qu et al., 2016; Wang et al., 2016, 2017). The level of reduction of the drive force, as well as the degree of stick-slip elimination, clearly depends on the amplitude $v_{a}$ of the velocity of the forced vibrations of the substrate and is higher if its value in relation to the speed of sliding is higher.

Abdo and Zaier (2012) reported that a significant impact on the reduction of stick-slip movement has displacement of the sliding specimen in the normal direction to the slip plane, which temporarily reduces the normal pressure at the contact. However, this problem was not analysed in this article according to the main topic because its explanation requires more extensive experimental tests of the possibility of creating normal vibration in sliding motion by the introduction of tangential vibration into the contact zone.

\section{SUMMARY}

The test stand presented in this article allows to conduct a wide range of experimental studies on the influence of longitudinal tangential vibrations on the stick-slip phenomenon occurring in sliding motion. The change of test variant is connected with the possibility to change the drive speed $v_{d}$, the frequency $f$ and amplitude $u_{0}$ of the forced vibrations, the external load $F_{z}$ and the stiffness of the drive $k_{d}$. The results of the tests presented in this article are only a selected fragment of one of the possible variants of the test.

The conducted experimental analyses have shown that longitudinal tangential vibrations of the substrate on which the slip movement is performed may contribute to the reduction or even complete elimination of the stick-slip phenomenon. The decisive parameter for partial or total elimination of this phenomenon is the value of amplitude of the velocity of vibrations in relation to the nominal speed of movement of the bodies in contact.

For values of $v_{d}, k_{d}, F_{z}$ and $f$, assumed in this stage of experimental research, in the range where the amplitude of the vibration velocity is lower than the drive velocity, only a gradual reduction of the stick-slip phenomenon occurs. As the $v_{a}$ value increases, the jump length of the sliding body decreases. If the amplitude of the vibration velocity is equal to or higher than the drive velocity, the 
stick-slip phenomenon is completely eliminated. An additional, observable effect in the range for $v_{a}$ higher than $v_{d}$ is the reduction of the drive force, where the higher the value of $v_{a}$ is, the greater is the reduction of this force.

\section{REFERENCES}

1. Abdo J., Tahat M., Abouelsoud A. (2009), The effect of excitation frequencies on stick-slip amplitude, 3rd International Conference on Integrity, Reliability and Failure, Porto/Portugal, 319-320.

2. Abdo J., Tahat M., Abouelsoud A., Danish M. (2010), The effect of frequency of vibration and humidity on the stick-slip amplitude, International Journal of Mechanics and Materials in Design, 6(1), 45-51.

3. Abdo J., Zaier R. (2012), A novel pin-on-disk machine for stick-slip measurements, Materials and Manufacturing Processes, 27, 751755.

4. Canudas de Wit C., Olsson H., Aström K.J., Lischinsky P. (1995), A new model for control of system with frictio, IEEE Transactions on Automatic Control, 40(3), 419-425.

5. F. Aarsnes U.J., Di Meglio F., Shor R.J. (2018), Avoiding stick slip vibrations in drilling through startup trajectory design, Journal of Process Control, 70, 24-35.

6. Gao H., De Volder M., Cheng T., Bao G., Reynaerts D. (2016), A pneumatic actuator based on vibration friction reduction with bending longitudinal vibration mode, Sensors and Actuators A: Physical, 252, 112-119.

7. Grudziński K., Warda B.J. (1993), A study of the influence of chosen tribological factors on the mixed friction characteristics and stick-slip vibrations, Archives of Mechanical Technology and Automation, 12, 499-514 (in Polish).

8. Gutowski P., Leus M. (2012), The effect of longitudinal tangential vibrations on friction and driving forces in sliding motion, Tribology International, 55, 108-118.

9. Gutowski P., Leus M. (2015), Computational model for friction force estimation in sliding motion at transverse tangential vibrations of elastic contact support. Tribology International, 90, 455-462.

10. Kapelke S., Seemann W. (2018), On the effect of longitudinal vibrations on dry friction: Modelling aspects and experimental investigations, Tribology Letters, 66(3), 1-11.

11. Kligerman Y., Varenberg M. (2014), Elimination of stick-slip motion in sliding of split or rough surface, Tribology Letters, 53(2), 395-399.

12. Kröger M., Neubauer M., Popp K. (2008), Experimental investigation on the avoidance of self-excited vibrations, Philosophical Transactions of the Royal Society A: Mathematical, Physical and Engineering Sciences, 366(1866), 785-810.

13. Leus M., Gutowski P. (2011), Practical possibilities of utilization of tangential longitudinal vibrations for controlling the friction force and reduction of drive force in sliding motion, Mechanics and Mechanical Engineering, 15(4), 103-113.

14. Littmann W., Stork H., Wallaschek J. (2001a), Reduction of friction using piezoelectrically excited ultrasonic vibrations, Proceedings of the SPIE's 8th Annual International Symposium on Smart Structures and Material, Billingham, Washington, 302-311.
15. Littmann W., Stork H., Wallaschek J. (2001b), Sliding friction in the presence of ultrasonic oscillations: superposition of longitudinal oscillations. Archive of Applied Mechanics, 71, 549-54.

16. Mfoumou G.S., Kenmoé G.D., Kofané T.C. (2019), Computational algorithms of time series for stick-slip dynamics and time-delayed feedback control of chaos for a class of discontinuous friction systems, Mechanical Systems and Signal Processing, 119, 399-419.

17. Mokhtar M.O.A., Younes Y.K., El Mahdy T.H., Attia N.A. (1998), A theoretical and experimental study on dynamics of sliding bodies with dry conformal contacts, Wear, 218(2), 172-178.

18. Neubauer M., Neuber C-C., Popp K. (2005), Control of stick-slip vibrations, Solid Mechanics and its Applications, 130, 223-232.

19. Ozaki S., Hashiguchi K. (2010), Numerical analysis of stick-slip instability by a rate-dependent elastoplastic formulation for friction, Tribology International, 43, 2120-2133.

20. Popov V.L., Starcevic J., Filippov, A.E. (2010), Influence of ultrasonic in-plane oscillations on static and sliding friction and intrinsic length scale of dry friction processes, Tribology Letters, 39(1), 25-30.

21. Popp K., Rudolph M. (2003), Avoidance of stick-slip motion by vibration control, Proceedings in Applied Mathematics and Mechanics, 3, 120-121.

22. Popp K., Rudolph M. (2004), Vibration control to avoid stick-slip motion, Journal of Vibration and Control, 10, 1585-1600.

23. Qiu H., Yang J., Butt S. (2018), Investigation on bit stick-slip vibration with random friction coefficients, Journal of Petroleum Science and Engineering, 164, 127-139.

24. Qu H., Zhou N., Guo W., Qu J. (2016), A model of friction reduction with in-plane high-frequency vibration, Proceedings of the Institution of Mechanical Engineers, Part J: Journal of Engineering Tribology, 230(8), 962-967.

25. Rymuza Z. (1992), The stick-slip phenomenon, PAK, 12, 290-295, (in Polish).

26. Tang L., Zhu X., Qian X., Shi C. (2017), Effects of weight on bit on torsional stick-slip vibration of oilwell drill string, Journal of Mechanical Science and Technology, 31(10), 4589-4597.

27. Tang L., Zhu X., Shi C., Tang J., Xu D. (2015), Study of the influences of rotary table speed on stick-slip vibration of the drilling system Petroleum, 1(4), 382-387.

28. Teidelt E., Starcevic J., Popov V.L. (2012), Influence of ultrasonic oscillation on static and sliding friction, Tribology Letters, 48(1), 51-62

29. Wang P., Ni H., Wang R., Li Z., Wang Y. (2016), Experimental investigation of the effect of in-plane vibrations on friction for different materials, Tribology International, 99, 237-247.

30. Wang P., Ni H., Wang R., Liu W., Lu S. (2017), Research on the mechanism of in-plane vibration on friction reduction, Materials, 10(9), 1-21.

31. Zhu X., Tang L., Yang Q. (2014), A literature review of approaches for stick-slip vibration suppression in oilwell drillstring, Advances in Mechanical Engineering, 2014, 967952, 1-17.

32. Zuleeg J. (2015), How to measure, prevent, and eliminate stick-slip and noise generation with lubricants, SAE Technical Paper 2015-01$2259,1-7$. 